\section{PTU-084 BARRETT'S OESOPHAGUS: A QUALITATIVE STUDY OF PATIENT BURDEN AND FOLLOW UP NEEDS}

1,2 James Britton*, 2,3 Shaheen Hamdy, ${ }^{2,3}$ John Mclaughlin, ${ }^{4}$ Maria Horne, ${ }^{2,3}$ Yeng Ang ${ }^{1}$ Wrightington, Wigan and Leigh NHS Trust, Leigh, UK; ${ }^{2}$ The University of Manchester, Manchester, UK; ${ }^{3}$ Salford Royal Foundation Trust, Salford, UK; ${ }^{4}$ The University of Leeds, Leeds, UK

\subsection{6/gutjnl-2018-BSGAbstracts.462}

Introduction The rising incidence of Barrett's oesophagus (BO) has implications on both service provision and patient burden. Few studies have assessed the impact on patients or engaged them in the design of care pathways. This qualitative study aims to: 1) identify factors impacting on BO patients' health-related quality of life; 2) explore their follow up needs and attitudes to new models of follow up care.

Methods An exploratory qualitative approach was utilised using 20 semi-structured, in-depth one-to-one interviews, audio recorded and transcribed verbatim. Patients undergoing BO surveillance at a single district general hospital were recruited using purposive sampling with the aim of achieving maximum variation in terms of age, sex and disease duration. Interviews focused on the impact of surveillance, physical and psychological symptoms, experiences of follow-up care, follow-up needs and new models of follow-up care. New models of care included a dedicated BO service and patient-initiated consultation by means of telephone or virtual clinic. Data were analysed using Framework approach, supported by NVivo Pro 11. Results Data saturation occurred after 20 participant interviews. Median age was 63 years (range $42-77$ years) and median disease duration was 5.6 years (range 1-15 years). 10 subthemes and three main themes emerged from data analysis: 1) Burden of disease symptom control, worry of oesophageal cancer and surveillance endoscopy; 2) Follow up experiences - historic and current follow up care, at this DGH, was found to be inconsistent and often inadequate to meet patients' needs and expectations. In particular, a lack of disease specific information; 3) Follow up needs - participants sought enhanced communication, organisation and structure of care. They valued face to face interaction with a specialist and were more likely to follow their advice. The concept of direct secondary care access in-between endoscopies was reassuring to participants. There was a strong preference towards a telephone patient-initiated consultation over an 'impersonal' online system.

Conclusions This qualitative research provides an in-depth account of the patients' perspective of BO in an NHS setting. The potential burden to patients must be considered when implementing future care pathways or when designing a BO specific patient reported outcome measure. To improve patient experiences we recommend the implementation and prospective assessment of a complex care intervention, which encompasses dedicated BO surveillance, outpatient clinic and telephone direct access line.

\section{PTU-085 A DEDICATED SERVICE IMPROVES THE ACCURACY OF BARRETT'S OESOPHAGUS SURVEILLANCE: A PROSPECTIVE COMPARATIVE COHORT STUDY}

\footnotetext{
1,2 James Britton*, ${ }^{3}$ Kelly Chatten, ${ }^{4}$ Thomas Riley, ${ }^{1}$ Alastair Cairns, ${ }^{1}$ Neeraj Prasad, ${ }^{1}$ Richard Keld, ${ }^{4,2}$ Shaheen Hamdy, ${ }^{4,2}$ John Mclaughlin, 4,2 Yeng Ang. 'Wrightington, Wigan and Leigh NHS Trust, Leigh, UK; ${ }^{2}$ The University of Manchester, Manchester, UK; ${ }^{3}$ Stockport NHS Foundation Trust, Stockport, UK; ${ }^{4}$ Salford Royal Foundation Trust, Salford, UK
}

Introduction This study aims to assess the quality of current Barrett's Oesophagus surveillance delivery against a dedicated service in the post BSG guideline era.

Methods All patients undergoing BO surveillance between January 2016 and July 2017 at a single NHS district general hospital (DGH) were included. Patients had their endoscopy conducted on a dedicated BO endoscopy list or a generic service list. Data were collected prospectively against the BSG guidelines. Prospective surveillance data were also compared to each patient's prior surveillance endoscopy experience.

Results 361 patients were scheduled for surveillance of which 217 attended a dedicated list (29 discharged, 13.4\%), 78 attended a non-dedicated list (7 discharged, 9\%) and 66 did not have their endoscopy. The cohorts were comparable in terms of age, sex and co-morbidity prevalence. The dedicated list adhered more closely to the BSG guidelines (table 1). Histology results from the dedicated list cohort revealed higher rates of intestinal metaplasia $(79.8 \%$ vs $73.1 \%, \mathrm{p}=0.1155)$ and dysplasia/OAC $(4.3 \%$ vs $2.6 \%, \mathrm{p}=0.4082)$ when compared to the non-dedicated, although statistical significance was not reached.

\begin{tabular}{|c|c|c|c|c|c|}
\hline BSG Standards & $\begin{array}{l}\text { Dedicated } \\
\text { BO } \\
\text { endoscopy } \\
(n=188)^{\wedge}\end{array}$ & $\begin{array}{l}\text { Non- } \\
\text { dedicated } \\
\text { endoscopy } \\
(\mathrm{n}=71)^{\wedge}\end{array}$ & $\begin{array}{l}\text { P value } \\
\text { Dedicated } \\
\text { vs Non- } \\
\text { dedicated }\end{array}$ & $\begin{array}{l}\text { Prior } \\
\text { endoscopy } \\
\text { (post BSG } \\
\text { guideline, } n= \\
229 \text { ) }\end{array}$ & $\begin{array}{l}\text { P Value } \\
\text { Dedicated } \\
\text { vs Prior }\end{array}$ \\
\hline Prague Classification & $\begin{array}{l}100 \% \\
(n=188)\end{array}$ & $\begin{array}{l}87.3 \% \\
(n=62)\end{array}$ & $P<0.0001$ & $\begin{array}{l}82.5 \% \\
(n=189)\end{array}$ & $P<0.0001$ \\
\hline $\begin{array}{l}\text { Barrett's island } \\
\text { description }\end{array}$ & $\begin{array}{l}96.6 \% \\
(n=28 / 29)\end{array}$ & $0 \%(n=0)$ & $P<0.0001$ & $\begin{array}{l}17.6 \%(n=3 / \\
17)\end{array}$ & $P<0.0001$ \\
\hline $\begin{array}{l}\text { Hiatus hernia } \\
\text { delineation }\end{array}$ & $\begin{array}{l}100 \% \\
(n=188)\end{array}$ & $\begin{array}{l}64.8 \% \\
(n=46)\end{array}$ & $P<0.0001$ & $\begin{array}{l}63.3 \% \\
(n=145)\end{array}$ & $P<0.0001$ \\
\hline $\begin{array}{l}\text { Visible lesion } \\
\text { documentation(yes or } \\
\text { no) }\end{array}$ & $\begin{array}{l}100 \% \\
(n=188)\end{array}$ & $\begin{array}{l}94.4 \% \\
(n=67)\end{array}$ & $P=0.0053$ & $\begin{array}{l}89.9 \% \\
(n=206)\end{array}$ & $P<0.0001$ \\
\hline $\begin{array}{l}\text { Visible lesion } \\
\text { description(distance } \\
\text { from incisors + Paris } \\
\text { classification) }\end{array}$ & $\begin{array}{l}94.4 \% \\
(n=17 / 18)\end{array}$ & $0 \%(n=0 / 6)$ & $P<0.0001$ & $0 \%(n=0 / 11)$ & $\mathrm{P}<0.0001$ \\
\hline $\begin{array}{l}\text { Biopsy } \\
\text { documentation } \\
\text { (location and } n \text { ) }\end{array}$ & $\begin{array}{l}99.5 \% \\
(n=187)\end{array}$ & $\begin{array}{l}5.6 \% \\
(n=4)\end{array}$ & $P<0.0001$ & $6.9 \%(n=16)$ & $P<0.0001$ \\
\hline $\begin{array}{l}\text { Seattle Protocol } \\
\text { Adherence }\end{array}$ & $\begin{array}{l}72 \% \\
(n=135 / \\
188)\end{array}$ & $\begin{array}{l}42 \% \\
(n=26 / 62)\end{array}$ & $P<0.0001$ & $\begin{array}{l}50 \%(n=94 / \\
189)\end{array}$ & $P<0.0001$ \\
\hline $\begin{array}{l}\text { Surveillance Interval } \\
\text { Adherence }\end{array}$ & $\begin{array}{l}100 \% \\
(n=188)\end{array}$ & * & * & $75 \%(n=147)$ & $P<0.0001$ \\
\hline
\end{tabular}

Conclusions The post-BSG guideline era of $\mathrm{BO}$ surveillance remains suboptimal in this DGH. A dedicated service can improve the accuracy and consistency of surveillance care pathways in line with current best practice, although the clinical significance of this remains to be determined.

\section{PTU-086 IMPROVING ENDOSCOPY EFFICIENCY BY REDUCING TURNAROUND TIME BETWEEN CASES}

Kathleen Bryce* ${ }^{*}$ Robert Fearn, Sam Murray. Homerton Hospital, London, UK 